\title{
An extension of a multidimensional Hilbert-type inequality
}

Jianhua Zhong and Bicheng Yang*

"Correspondence:
bcyang@gdei.edu.cn
Department of Mathematics,
Guangdong University of
Education, Guangzhou, Guangdong
510303, P.R. China

510303, P.R. China

\begin{abstract}
In this paper, by the use of weight coefficients, the transfer formula and the technique of real analysis, a new multidimensional Hilbert-type inequality with multi-parameters and a best possible constant factor is given, which is an extension of some published results. Moreover, the equivalent forms, the operator expressions and a few particular inequalities are considered.
\end{abstract}

MSC: 26D15; 47A05

Keywords: Hilbert-type inequality; weight coefficient; equivalent form; operator; norm

\section{Introduction}

If $p>1, \frac{1}{p}+\frac{1}{q}=1, a_{m}, b_{n} \geq 0, a=\left\{a_{m}\right\}_{m=1}^{\infty} \in l^{p}, b=\left\{b_{n}\right\}_{n=1}^{\infty} \in l^{q},\|a\|_{p}=\left(\sum_{m=1}^{\infty} a_{m}^{p}\right)^{\frac{1}{p}}>0$, $\|b\|_{q}>0$, then we have the following Hardy-Hilbert inequality with the best possible constant $\frac{\pi}{\sin (\pi / p)}$ :

$$
\sum_{m=1}^{\infty} \sum_{n=1}^{\infty} \frac{a_{m} b_{n}}{m+n}<\frac{\pi}{\sin (\pi / p)}\|a\|_{p}\|b\|_{q}
$$

and the following Hilbert-type inequality:

$$
\sum_{m=1}^{\infty} \sum_{n=1}^{\infty} \frac{a_{m} b_{n}}{\max \{m, n\}}<p q\|a\|_{p}\|b\|_{q}
$$

with the best possible constant factor $p q$ (cf. [1], Theorem 315, Theorem 341). Inequalities (1) and (2) are important in the analysis and its applications (cf. [1-3]).

Assuming that $\left\{\mu_{m}\right\}_{m=1}^{\infty},\left\{v_{n}\right\}_{n=1}^{\infty}$ are positive sequences,

$$
U_{m}=\sum_{i=1}^{m} \mu_{i}, \quad V_{n}=\sum_{j=1}^{n} v_{j} \quad(m, n \in \mathbb{N}=\{1,2, \ldots\}),
$$

we have the following Hardy-Hilbert-type inequality (cf. [1], Theorem 321):

$$
\sum_{m=1}^{\infty} \sum_{n=1}^{\infty} \frac{a_{m} b_{n}}{U_{m}+V_{n}}<\frac{\pi}{\sin (\pi / p)}\left(\sum_{m=1}^{\infty} \frac{a_{m}^{p}}{m^{p-1}}\right)^{\frac{1}{p}}\left(\sum_{n=1}^{\infty} \frac{b_{n}^{q}}{n^{q-1}}\right)^{\frac{1}{q}} .
$$

(c) The Author(s) 2017. This article is distributed under the terms of the Creative Commons Attribution 4.0 International License (http://creativecommons.org/licenses/by/4.0/), which permits unrestricted use, distribution, and reproduction in any medium, provided you give appropriate credit to the original author(s) and the source, provide a link to the Creative Commons license, and indicate if changes were made. 
For $\mu_{i}=v_{j}=1(i, j \in \mathbb{N})$, inequality (3) reduces to (1).

In 2014, Yang and Chen [4] gave the following multidimensional Hilbert-type inequality: For $i_{0}, j_{0} \in \mathbb{N}, \alpha, \beta>0$,

$$
\begin{aligned}
& \|x\|_{\alpha}:=\left(\sum_{k=1}^{i_{0}}\left|x^{(k)}\right|^{\alpha}\right)^{\frac{1}{\alpha}} \quad\left(x=\left(x^{(1)}, \ldots, x^{\left(i_{0}\right)}\right) \in \mathbb{R}^{i_{0}}\right), \\
& \|y\|_{\beta}:=\left(\sum_{k=1}^{j_{0}}\left|y^{(k)}\right|^{\beta}\right)^{\frac{1}{\beta}} \quad\left(y=\left(y^{(1)}, \ldots, y^{\left(j_{0}\right)}\right) \in \mathbb{R}^{j_{0}}\right),
\end{aligned}
$$

$0<\lambda_{1}+\eta \leq i_{0}, 0<\lambda_{2}+\eta \leq j_{0}, \lambda_{1}+\lambda_{2}=\lambda, a_{m}, b_{n} \geq 0$, we have

$$
\begin{aligned}
& \sum_{n} \sum_{m} \frac{\left(\min \left\{\|m\|_{\alpha},\|n\|_{\beta}\right\}\right)^{\eta}}{\left(\max \left\{\|m\|_{\alpha},\|n\|_{\beta}\right\}\right)^{\lambda+\eta}} a_{m} b_{n} \\
&<K_{1}^{\frac{1}{p}} K_{2}^{\frac{1}{q}}\left[\sum_{m}\|m\|_{\alpha}^{p\left(i_{0}-\lambda_{1}\right)-i_{0}} a_{m}^{p}\right]^{\frac{1}{p}}\left[\sum_{n}\|n\|_{\beta}^{q\left(j_{0}-\lambda_{2}\right)-j_{0}} b_{n}^{q}\right]^{\frac{1}{q}},
\end{aligned}
$$

where $\sum_{m}=\sum_{m_{i_{0}}=1}^{\infty} \cdots \sum_{m_{1}=1}^{\infty}, \sum_{n}=\sum_{n_{j_{0}}=1}^{\infty} \cdots \sum_{n_{1}=1}^{\infty}$, the series on the right-hand side are positive, and the best possible constant factor $K_{1}^{\frac{1}{p}} K_{2}^{\frac{1}{q}}$ is indicated by

$$
K_{1}^{\frac{1}{p}} K_{2}^{\frac{1}{q}}=\left[\frac{\Gamma^{j_{0}}\left(\frac{1}{\beta}\right)}{\beta^{j_{0}-1} \Gamma\left(\frac{j_{0}}{\beta}\right)}\right]^{\frac{1}{p}}\left[\frac{\Gamma^{i_{0}}\left(\frac{1}{\alpha}\right)}{\alpha^{i_{0}-1} \Gamma\left(\frac{i_{0}}{\alpha}\right)}\right]^{\frac{1}{q}} \frac{\lambda+2 \eta}{\left(\lambda_{1}+\eta\right)\left(\lambda_{2}+\eta\right)} .
$$

For $i_{0}=j_{0}=\lambda=1, \eta=0, \lambda_{1}=\frac{1}{q}, \lambda_{2}=\frac{1}{p}$, inequality (4) reduces to (2). The other results on this type of inequalities were provided by [5-17].

In 2015, Shi and Yang [18] gave another extension of (2) as follows:

$$
\sum_{m=1}^{\infty} \sum_{n=1}^{\infty} \frac{a_{m} b_{n}}{\max \left\{U_{m}, V_{n}\right\}}<p q\left(\sum_{m=1}^{\infty} \frac{a_{m}^{p}}{m^{p-1}}\right)^{\frac{1}{p}}\left(\sum_{n=1}^{\infty} \frac{b_{n}^{q}}{n^{q-1}}\right)^{\frac{1}{q}} .
$$

Some other results on Hardy-Hilbert-type inequalities were given by [19-25].

In this paper, by the use of weight coefficients, the transfer formula and the technique of real analysis, a new multidimensional Hilbert-type inequality with multi-parameters and a best possible constant factor is given, which is an extension of (4) and (5). Moreover, the equivalent forms, the operator expressions and a few particular inequalities are considered.

\section{Some lemmas}

If $\mu_{i}^{(k)}>0\left(k=1, \ldots, i_{0} ; i=1, \ldots, m\right), v_{j}^{(l)}>0\left(l=1, \ldots, j_{0} ; j=1, \ldots, n\right)$, then we set

$$
\begin{aligned}
& U_{m}^{(k)}:=\sum_{i=1}^{m} \mu_{i}^{(k)} \quad\left(k=1, \ldots, i_{0}\right), \quad V_{n}^{(l)}:=\sum_{j=1}^{n} v_{j}^{(l)} \quad\left(l=1, \ldots, j_{0}\right), \\
& U_{m}=\left(U_{m}^{(1)}, \ldots, U_{m}^{\left(i_{0}\right)}\right), \quad V_{n}=\left(V_{n}^{(1)}, \ldots, V_{n}^{\left(j_{0}\right)}\right) \quad(m, n \in \mathbb{N}) .
\end{aligned}
$$

We also set functions $\mu_{k}(t):=\mu_{m}^{(k)}, t \in(m-1, m](m \in \mathbb{N}) ; v_{l}(t):=v_{n}^{(l)}, t \in(n-1, n](n \in \mathbb{N})$, and 


$$
\begin{aligned}
& U_{k}(x):=\int_{0}^{x} \mu_{k}(t) d t \quad\left(k=1, \ldots, i_{0}\right), \\
& V_{l}(y):=\int_{0}^{y} v_{l}(t) d t \quad\left(l=1, \ldots, j_{0}\right), \\
& U(x):=\left(U_{1}(x), \ldots, U_{i_{0}}(x)\right), \quad V(y):=\left(V_{1}(y), \ldots, V_{j_{0}}(y)\right) \quad(x, y \geq 0) .
\end{aligned}
$$

It follows that $U_{k}(m)=U_{m}^{(k)}\left(k=1, \ldots, i_{0} ; m \in \mathbb{N}\right), V_{l}(n)=V_{n}^{(l)}\left(l=1, \ldots, j_{0} ; n \in \mathbb{N}\right)$, and for $x \in(m-1, m), U_{k}^{\prime}(x)=\mu_{k}(x)=\mu_{m}^{(k)}\left(k=1, \ldots, i_{0} ; m \in \mathbb{N}\right) ;$ for $y \in(n-1, n), V_{l}^{\prime}(y)=v_{l}(y)=v_{n}^{(l)}$ $\left(l=1, \ldots, j_{0} ; n \in \mathbb{N}\right)$.

Lemma 1 (cf. [21]) Suppose that $g(t)(>0)$ is decreasing in $\mathbb{R}_{+}$and strictly decreasing in $\left[n_{0}, \infty\right)\left(n_{0} \in \mathbb{N}\right)$, satisfying $\int_{0}^{\infty} g(t) d t \in \mathbb{R}_{+}$. We have

$$
\int_{1}^{\infty} g(t) d t<\sum_{n=1}^{\infty} g(n)<\int_{0}^{\infty} g(t) d t
$$

Lemma 2 If $i_{0} \in \mathbb{N}, \alpha, M>0, \Psi(u)$ is a non-negative measurable function in $(0,1]$, and

$$
D_{M}:=\left\{x \in \mathbb{R}_{+}^{i_{0}} ; u=\sum_{i=1}^{i_{0}}\left(\frac{x_{i}}{M}\right)^{\alpha} \leq 1\right\},
$$

then we have the following transfer formula (cf. [26]):

$$
\int \cdots \int_{D_{M}} \Psi\left(\sum_{i=1}^{i_{0}}\left(\frac{x_{i}}{M}\right)^{\alpha}\right) d x_{1} \cdots d x_{s}=\frac{M^{i_{0}} \Gamma^{i_{0}}\left(\frac{1}{\alpha}\right)}{\alpha^{i_{0}} \Gamma\left(\frac{i_{0}}{\alpha}\right)} \int_{0}^{1} \Psi(u) u^{\frac{i_{0}}{\alpha}-1} d u .
$$

Lemma 3 For $i_{0}, j_{0} \in \mathbb{N}, \mu_{m}^{(k)} \geq \mu_{m+1}^{(k)}\left(m \in \mathbb{N}, k=1, \ldots, i_{0}\right), v_{n}^{(l)} \geq v_{n+1}^{(l)}\left(n \in \mathbb{N} ; l=1, \ldots, j_{0}\right)$, $\alpha, \beta>0, \varepsilon>0$, we have

$$
\begin{aligned}
& \sum_{m}\left\|U_{m}\right\|_{\alpha}^{-i_{0}-\varepsilon} \prod_{k=1}^{i_{0}} \mu_{m}^{(k)} \leq \frac{\Gamma^{i_{0}}\left(\frac{1}{\alpha}\right)}{\varepsilon i_{0}^{\varepsilon^{\varepsilon / \alpha}} \alpha^{i_{0}-1} \Gamma\left(\frac{i_{0}}{\alpha}\right)}+O(1), \\
& \sum_{n}\left\|V_{n}\right\|_{\beta}^{-j_{0}-\varepsilon} \prod_{k=1}^{j_{0}} v_{n}^{(k)} \leq \frac{\Gamma^{j_{0}}\left(\frac{1}{\beta}\right)}{\varepsilon \xi_{0}^{\varepsilon / \beta} \beta^{j_{0}-1} \Gamma\left(\frac{j_{0}}{\beta}\right)}+\tilde{O}(1) \quad\left(\varepsilon \rightarrow 0^{+}\right) .
\end{aligned}
$$

Proof For $M>i_{0}^{1 / \alpha}$, we set

$$
\Psi(u)= \begin{cases}0, & 0<u<\frac{i_{0}}{M^{\alpha}}, \\ \frac{1}{\left(M u^{1 / \alpha}\right)^{i_{0}+\varepsilon}}, & \frac{i_{0}}{M^{\alpha}} \leq u \leq 1 .\end{cases}
$$

By (12), it follows that

$$
\begin{aligned}
\int_{\left\{x \in \mathbb{R}_{+}^{i_{0}} ; x_{i} \geq 1\right\}} \frac{d x}{\|x\|_{\alpha}^{i_{0}+\varepsilon}} & =\lim _{M \rightarrow \infty} \int \cdots \int_{D_{M}} \Psi\left(\sum_{i=1}^{i_{0}}\left(\frac{x_{i}}{M}\right)^{\alpha}\right) d x_{1} \cdots d x_{i_{0}} \\
& =\lim _{M \rightarrow \infty} \frac{M^{i_{0}} \Gamma^{i_{0}}\left(\frac{1}{\alpha}\right)}{\alpha^{i_{0}} \Gamma\left(\frac{i_{0}}{\alpha}\right)} \int_{i_{0} / M^{\alpha}}^{1} \frac{u^{i_{0}}-1}{\left(M u^{1 / \alpha}\right)^{i_{0}+\varepsilon}} d u=\frac{\Gamma^{i_{0}}\left(\frac{1}{\alpha}\right)}{\varepsilon i_{0}^{\varepsilon / \alpha} \alpha^{i_{0}-1} \Gamma\left(\frac{i_{0}}{\alpha}\right)} .
\end{aligned}
$$


Then by (10) and the above result, we find

$$
\begin{aligned}
0< & \sum_{\left\{m \in \mathbb{N}^{i_{0}} ; m_{i} \geq 2\right\}}\left\|U_{m}\right\|_{\alpha}^{-i_{0}-\varepsilon} \prod_{k=1}^{i_{0}} \mu_{m}^{(k)} \\
= & \sum_{\left\{m \in \mathbb{N}_{0}^{i} ; m_{i} \geq 2\right\}} \int_{\left\{x \in \mathbb{N}^{i_{0}} ; m-1 \leq x_{i}<m\right\}}\|U(m)\|_{\alpha}^{-i_{0}-\varepsilon} \prod_{k=1}^{i_{0}} \mu_{m}^{(k)} d x \\
< & \sum_{\left\{m \in \mathbb{N}^{i_{0}} ; m_{i} \geq 2\right\}} \int_{\left\{x \in \mathbb{N}^{i_{0}} ; m-1 \leq x_{i}<m\right\}}\|U(x)\|_{\alpha}^{-i_{0}-\varepsilon} \prod_{k=1}^{i_{0}} \mu_{m}^{(k)}(x) d x \\
= & \int_{\left\{x \in \mathbb{N}^{i_{0}} ; x_{i} \geq 1\right\}}\|U(x)\|_{\alpha}^{-i_{0}-\varepsilon} \prod_{k=1}^{i_{0}} \mu^{(k)}(x) d x^{\nu=U}=\int_{\left\{v \in \mathbb{R}_{+}^{i_{0}} ; v_{i} \geq \mu_{1}^{(i)}\right\}}\|\nu\|_{\alpha}^{-i_{0}-\varepsilon} d \nu \\
= & \int_{\left\{v \in \mathbb{R}_{+}^{i_{0}} ; v_{i} \geq 1\right\}}\|\nu\|_{\alpha}^{-i_{0}-\varepsilon} d \nu+O_{i_{0}}(1)=\frac{\Gamma^{i_{0}}\left(\frac{1}{\alpha}\right)}{\varepsilon i_{0}^{\varepsilon / \alpha} \alpha^{i_{0}-1} \Gamma\left(\frac{i_{0}}{\alpha}\right)}+O_{i_{0}}(1) .
\end{aligned}
$$

For $i_{0}=1,0<\sum_{\left\{m \in \mathbb{N}^{i} 0 ; m_{i}=1\right\}}\left\|U_{m}\right\|_{\alpha}^{-i_{0}-\varepsilon} \prod_{k=1}^{i_{0}} \mu_{m}^{(k)}<\infty$; for $i_{0} \geq 2, \mu^{(i)}=\max _{m} \mu_{m}^{(i)}, b=$ $\sum_{i=1}^{i_{0}} \mu^{(i)}$, in the same way, we find

$$
\begin{aligned}
0 & <\sum_{\left\{m \in \mathbb{N}^{i} 0 ; \exists i, m_{i}=1\right\}}\left\|U_{m}\right\|_{\alpha}^{-i_{0}-\varepsilon} \prod_{k=1}^{i_{0}} \mu_{m}^{(k)} \\
& \leq\left\|U_{1}\right\|_{\alpha}^{-i_{0}-\varepsilon} \prod_{k=1}^{i_{0}} \mu_{1}^{(k)}+\sum_{i=1}^{i_{0}} \mu^{(i)} \sum_{\left\{m \in \mathbb{N}^{i_{0}-1} ; m_{j} \geq 2(j \neq i)\right\}}\left\|U_{m}\right\|_{\alpha}^{-\left(i_{0}-1\right)-(\varepsilon+1)} \prod_{k=1(k \neq i)}^{i_{0}} \mu_{m}^{(k)} \\
& =O_{1}(1)+\frac{b \Gamma^{i_{0}-1}\left(\frac{1}{\alpha}\right)}{(1+\varepsilon)\left(i_{0}-1\right)^{(1+\varepsilon) / \alpha} \alpha^{i_{0}-2} \Gamma\left(\frac{i_{0}-1}{\alpha}\right)}+b O_{i_{0}-1}(1)<\infty .
\end{aligned}
$$

Then we have

$$
\begin{aligned}
\sum_{m}\left\|U_{m}\right\|_{\alpha}^{-i_{0}-\varepsilon} \prod_{k=1}^{i_{0}} \mu_{m}^{(k)}= & \sum_{\left\{m \in \mathbb{N}_{0}^{i} ; \exists i, m_{i}=1\right\}} \sum_{m}\left\|U_{m}\right\|_{\alpha}^{-i_{0}-\varepsilon} \prod_{k=1}^{i_{0}} \mu_{m}^{(k)} \\
& +\sum_{\left\{m \in \mathbb{N}^{i_{0}} ; m_{j} \geq 2\right\}}\left\|U_{m}\right\|_{\alpha}^{-i_{0}-\varepsilon} \prod_{k=1}^{i_{0}} \mu_{m}^{(k)} \\
\leq & \frac{\Gamma^{i_{0}}\left(\frac{1}{\alpha}\right)}{\varepsilon i_{0}^{\varepsilon / \alpha} \alpha^{i_{0}-1} \Gamma\left(\frac{i_{0}}{\alpha}\right)}+O(1) \quad\left(\varepsilon \rightarrow 0^{+}\right) .
\end{aligned}
$$

Hence, we have (13). In the same way, we have (14).

Definition 1 For $\alpha, \beta>0,0<\lambda_{1}+\eta \leq i_{0}, 0<\lambda_{2}+\eta \leq j_{0}, \lambda_{1}+\lambda_{2}=\lambda$, we define two weight coefficients $w\left(\lambda_{1}, n\right)$ and $W\left(\lambda_{2}, m\right)$ as follows:

$$
w\left(\lambda_{1}, n\right):=\sum_{m} \frac{\left(\min \left\{\left\|U_{m}\right\|_{\alpha},\left\|V_{n}\right\|_{\beta}\right\}\right)^{\eta}}{\left(\max \left\{\left\|U_{m}\right\|_{\alpha},\left\|V_{n}\right\|_{\beta}\right\}\right)^{\lambda+\eta}} \frac{\left\|V_{n}\right\|_{\beta}^{\lambda_{2}}}{\left\|U_{m}\right\|_{\alpha}^{i_{0}-\lambda_{1}}} \prod_{k=1}^{i_{0}} \mu_{m}^{(k)},
$$




$$
W\left(\lambda_{2}, m\right):=\sum_{n} \frac{\left(\min \left\{\left\|U_{m}\right\|_{\alpha},\left\|V_{n}\right\|_{\beta}\right\}\right)^{\eta}}{\left(\max \left\{\left\|U_{m}\right\|_{\alpha},\left\|V_{n}\right\|_{\beta}\right\}\right)^{\lambda+\eta}} \frac{\left\|U_{m}\right\|_{\alpha}^{\lambda_{1}}}{\left\|V_{n}\right\|_{\beta}^{j_{0}-\lambda_{2}}} \prod_{l=1}^{j_{0}} v_{n}^{(l)} .
$$

Example 1 With regard to the assumptions of Definition 1, we set

$$
k_{\lambda}(x, y)=\frac{(\min \{x, y\})^{\eta}}{(\max \{x, y\})^{\lambda+\eta}} \quad(x, y>0) .
$$

Then, (i) for fixed $y>0$,

$$
k_{\lambda}(x, y) \frac{1}{x^{i_{0}-\lambda_{1}}}= \begin{cases}\frac{1}{y^{\lambda+\eta} x^{i_{0}-\lambda_{1}-\eta}}, & 0<x<y, \\ \frac{y^{\eta}}{x^{i_{0}+\lambda_{2}+\eta}}, & x \geq y,\end{cases}
$$

is decreasing in $\mathbb{R}_{+}$and strictly decreasing in $([y]+1, \infty)$. In the same way, for fixed $x>0$, $k_{\lambda}(x, y) \frac{1}{y^{1} 0^{-\lambda_{2}}}$ is decreasing in $\mathbb{R}_{+}$and strictly decreasing in $([x]+1, \infty)$. We still have

$$
\begin{aligned}
k\left(\lambda_{1}\right) & :=\int_{0}^{\infty} k_{\lambda}(u, 1) \frac{d u}{u^{1-\lambda_{1}}}=\int_{0}^{\infty} \frac{(\min \{u, 1\})^{\eta}}{(\max \{u, 1\})^{\lambda+\eta}} \frac{d u}{u^{1-\lambda_{1}}} \\
& =\int_{0}^{1} \frac{u^{\eta}}{u^{1-\lambda_{1}}} d u+\int_{1}^{\infty} \frac{1}{u^{\lambda+\eta}} \frac{d u}{u^{1-\lambda_{1}}}=\frac{\lambda+2 \eta}{\left(\lambda_{1}+\eta\right)\left(\lambda_{2}+\eta\right)} .
\end{aligned}
$$

(ii) For $b>0$, we have

$$
\frac{d}{d x}\left(b+x^{\alpha}\right)^{\frac{1}{\alpha}}=\left(b+x^{\alpha}\right)^{\frac{1}{\alpha}-1} x^{\alpha-1}>0 \quad(x>0) .
$$

Hence, for $m-1<x_{i}<m\left(i=1, \ldots, i_{0} ; m \in \mathbb{N}\right)$, we have $\|U(m)\|_{\alpha}>\|U(x)\|_{\alpha}$ and

$$
\begin{aligned}
& \frac{\left(\min \left\{\|U(m)\|_{\alpha},\left\|V_{n}\right\|_{\beta}\right\}\right)^{\eta}}{\left(\max \left\{\|U(m)\|_{\alpha},\left\|V_{n}\right\|_{\beta}\right\}\right)^{\lambda+\eta}} \frac{1}{\|U(m)\|_{\alpha}^{i_{0}-\lambda_{1}}} \\
& <\frac{\left(\min \left\{\|U(x)\|_{\alpha},\left\|V_{n}\right\|_{\beta}\right\}\right)^{\eta}}{\left(\max \left\{\|U(x)\|_{\alpha},\left\|V_{n}\right\|_{\beta}\right\}\right)^{\lambda+\eta}} \frac{1}{\|U(x)\|_{\alpha}^{i_{0}-\lambda_{1}}} ;
\end{aligned}
$$

for $m<x_{i}<m+1\left(i=1, \ldots, i_{0} ; m \in \mathbb{N}\right)$, we have $\|U(m)\|_{\alpha}<\|U(x)\|_{\alpha}$ and

$$
\begin{aligned}
& \frac{\left(\min \left\{\|U(m)\|_{\alpha},\left\|V_{n}\right\|_{\beta}\right\}\right)^{\eta}}{\left(\max \left\{\|U(m)\|_{\alpha},\left\|V_{n}\right\|_{\beta}\right\}\right)^{\lambda+\eta}} \frac{1}{\|U(m)\|_{\alpha}^{i_{0}-\lambda_{1}}} \\
& >\frac{\left(\min \left\{\|U(x)\|_{\alpha},\left\|V_{n}\right\|_{\beta}\right\}\right)^{\eta}}{\left(\max \left\{\|U(x)\|_{\alpha},\left\|V_{n}\right\|_{\beta}\right\}\right)^{\lambda+\eta}} \frac{1}{\|U(x)\|_{\alpha}^{i_{0}-\lambda_{1}}} .
\end{aligned}
$$

Lemma 4 With regard to the assumptions of Definition 1, (i) we have

$$
\begin{aligned}
& w\left(\lambda_{1}, n\right)<K_{2}\left(\lambda_{1}\right) \quad\left(n \in \mathbb{N}^{j}\right), \\
& W\left(\lambda_{2}, m\right)<K_{1}\left(\lambda_{1}\right) \quad\left(m \in \mathbb{N}^{i_{0}}\right),
\end{aligned}
$$

where

$$
K_{1}\left(\lambda_{1}\right)=\frac{\Gamma^{j_{0}}\left(\frac{1}{\beta}\right)}{\beta^{j_{0}-1} \Gamma\left(\frac{j_{0}}{\beta}\right)} k\left(\lambda_{1}\right), \quad K_{2}\left(\lambda_{1}\right)=\frac{\Gamma^{i_{0}}\left(\frac{1}{\alpha}\right)}{\alpha^{i_{0}-1} \Gamma\left(\frac{i_{0}}{\alpha}\right)} k\left(\lambda_{1}\right) ;
$$


(ii) for $\mu_{m}^{(k)} \geq \mu_{m+1}^{(k)}(m \in \mathbb{N}), v_{n}^{(l)} \geq v_{n+1}^{(l)}(n \in \mathbb{N}), U_{\infty}^{(k)}=V_{\infty}^{(l)}\left(k=1, \ldots, i_{0}, l=1, \ldots, j_{0}\right), 0<$ $\lambda_{1}+\eta \leq i_{0}, \lambda_{2}+\eta>0,0<\varepsilon<p \lambda_{1}(p>1)$, we have

$$
0<K_{2}\left(\lambda_{1}\right)\left(1-\theta_{\lambda}(n)\right)<w\left(\lambda_{1}, n\right) \quad\left(n \in \mathbb{N}^{j 0}\right),
$$

where, for $c:=\max _{1 \leq k \leq i_{0}}\left\{\mu_{1}^{(k)}\right\}(>0)$,

$$
\theta_{\lambda}(n):=\frac{1}{k\left(\lambda_{1}\right)} \int_{0}^{c c_{0}^{1 / \alpha} /\left\|V_{n}\right\|_{\beta}} \frac{(\min \{v, 1\})^{\eta} v^{\lambda_{1}-1}}{(\max \{v, 1\})^{\lambda+\eta}} d v=O\left(\frac{1}{\left\|V_{n}\right\|_{\beta}^{\lambda_{1}+\eta}}\right) .
$$

Proof (i) By (10), (12) and Example 1(ii), for $0<\lambda_{1}+\eta \leq i_{0}, \lambda>0$, it follows that

$$
\begin{aligned}
& w\left(\lambda_{1}, n\right)=\sum_{m} \int_{\left\{x \in \mathbb{N}^{i} 0 ; m_{i}-1 \leq x_{i} \leq m_{i}\right\}} \frac{\left(\min \left\{\|U(m)\|_{\alpha},\left\|V_{n}\right\|_{\beta}\right\}\right)^{\eta}}{\left(\max \left\{\|U(m)\|_{\alpha},\left\|V_{n}\right\|_{\beta}\right\}\right)^{\lambda+\eta}} \\
& \times \frac{\left\|V_{n}\right\|_{\beta}^{\lambda_{2}}}{\|U(m)\|_{\alpha}^{i_{0}-\lambda_{1}}} \prod_{k=1}^{i_{0}} \mu_{m}^{(k)} d x \\
& <\sum_{m} \int_{\left\{x \in \mathbb{N}^{i} ; m_{i}-1 \leq x_{i} \leq m_{i}\right\}} \frac{\left(\min \left\{\|U(x)\|_{\alpha},\left\|V_{n}\right\|_{\beta}\right\}\right)^{\eta}}{\left(\max \left\{\|U(x)\|_{\alpha},\left\|V_{n}\right\|_{\beta}\right\}\right)^{\lambda+\eta}} \\
& \times \frac{\left\|V_{n}\right\|_{\beta}^{\lambda_{2}}}{\|U(x)\|_{\alpha}^{i_{0}-\lambda_{1}}} \prod_{k=1}^{i_{0}} \mu_{m}^{(k)}(x) d x \\
& =\int_{\mathbb{R}_{+}^{i_{0}}} \frac{\left(\min \left\{\|U(x)\|_{\alpha},\left\|V_{n}\right\|_{\beta}\right\}\right)^{\eta}}{\left(\max \left\{\|U(x)\|_{\alpha},\left\|V_{n}\right\|_{\beta}\right\}\right)^{\lambda+\eta}} \frac{\left\|V_{n}\right\|_{\beta}^{\lambda_{2}}}{\|U(x)\|_{\alpha}^{i_{0}-\lambda_{1}}} \prod_{k=1}^{i_{0}} \mu^{(k)}(x) d x \\
& \stackrel{u=U(x)}{\leq} \int_{\mathbb{R}_{+}^{i_{0}}} \frac{\left(\min \left\{\|u\|_{\alpha},\left\|V_{n}\right\|_{\beta}\right\}\right)^{\eta}}{\left(\max \left\{\|u\|_{\alpha},\left\|V_{n}\right\|_{\beta}\right\}\right)^{\lambda+\eta}} \frac{\left\|V_{n}\right\|_{\beta}^{\lambda_{2}}}{\|u\|_{\alpha}^{i_{0}-\lambda_{1}}} d u \\
& =\lim _{M \rightarrow \infty} \int_{D_{M}} \frac{\left(\min \left\{M\left[\sum_{i=1}^{i_{0}}\left(\frac{u_{i}}{M}\right)^{\alpha}\right]^{1 / \alpha},\left\|V_{n}\right\|_{\beta}\right\}\right)^{\eta}}{\left(\max \left\{M\left[\sum_{i=1}^{i_{0}}\left(\frac{u_{i}}{M}\right)^{\alpha}\right]^{1 / \alpha},\left\|V_{n}\right\|_{\beta}\right\}\right)^{\lambda+\eta}} \frac{M^{\lambda_{1}-i_{0}}\left\|V_{n}\right\|_{\beta}^{\lambda_{2}} d u}{\left[\sum_{i=1}^{i_{0}}\left(\frac{u_{i}}{M}\right)^{\alpha}\right]^{\left(i_{0}-\lambda_{1}\right) / \alpha}}
\end{aligned}
$$

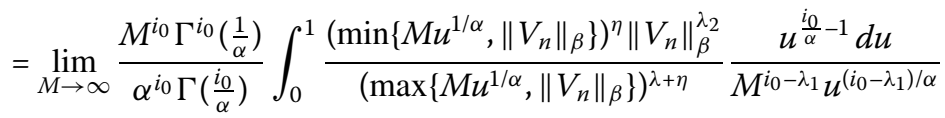

$$
\begin{aligned}
& =\lim _{M \rightarrow \infty} \frac{M^{i_{0}} \Gamma^{i_{0}}\left(\frac{1}{\alpha}\right)}{\alpha^{i_{0}} \Gamma\left(\frac{i_{0}}{\alpha}\right)} \int_{0}^{1} \frac{\left(\min \left\{M u^{1 / \alpha},\left\|V_{n}\right\|_{\beta}\right\}\right)^{\eta}\left\|V_{n}\right\|_{\beta}^{\lambda_{2}}}{\left(\max \left\{M u^{1 / \alpha},\left\|V_{n}\right\|_{\beta}\right\}\right)^{\lambda+\eta}} u^{\frac{\lambda_{1}}{\alpha}-1} d u \\
& \stackrel{v=\frac{M u^{1 / \alpha}}{\left\|V_{n}\right\|_{\beta}}}{=} \frac{\Gamma^{i_{0}}\left(\frac{1}{\alpha}\right)}{\alpha^{i_{0}-1} \Gamma\left(\frac{i_{0}}{\alpha}\right)} \int_{0}^{\infty} \frac{(\min \{v, 1\})^{\eta} v^{\lambda_{1}-1}}{(\max \{v, 1\})^{\lambda+\eta}} d v \\
& =\frac{\Gamma^{i_{0}}\left(\frac{1}{\alpha}\right)}{\alpha^{i_{0}-1} \Gamma\left(\frac{i_{0}}{\alpha}\right)} \frac{\lambda+2 \eta}{\left(\lambda_{1}+\eta\right)\left(\lambda_{2}+\eta\right)}=K_{2}\left(\lambda_{1}\right) .
\end{aligned}
$$

Hence, we have (18). In the same way, we have (19).

(ii) By (10) and in the same way, for $c=\max _{1 \leq k \leq i_{0}}\left\{\mu_{1}^{(k)}\right\}(>0)$, we have

$$
\begin{aligned}
w\left(\lambda_{1}, n\right) \geq & \sum_{m} \int_{\left\{x \in \mathbb{N}^{i} 0 ; m_{i} \leq x_{i} \leq m_{i}+1\right\}} \frac{\left(\min \left\{\|U(m)\|_{\alpha},\left\|V_{n}\right\|_{\beta}\right\}\right)^{\eta}}{\left(\max \left\{\|U(m)\|_{\alpha},\left\|V_{n}\right\|_{\beta}\right\}\right)^{\lambda+\eta}} \\
& \times \frac{\left\|V_{n}\right\|_{\beta}^{\lambda_{2}}}{\|U(m)\|_{\alpha}^{i_{0}-\lambda_{1}}} \prod_{k=1}^{i_{0}} \mu_{m+1}^{(k)} d x
\end{aligned}
$$




$$
\begin{aligned}
& >\sum_{m} \int_{\left\{x \in \mathbb{N}_{0}^{i_{0}} ; m_{i} \leq x_{i} \leq m_{i}+1\right\}} \frac{\left(\min \left\{\|U(x)\|_{\alpha},\left\|V_{n}\right\|_{\beta}\right\}\right)^{\eta}}{\left(\max \left\{\|U(x)\|_{\alpha},\left\|V_{n}\right\|_{\beta}\right\}\right)^{\lambda+\eta}} \\
& \quad \times \frac{\left\|V_{n}\right\|_{\beta}^{\lambda_{2}}}{\|U(x)\|_{\alpha}^{i_{0}-\lambda_{1}}} \prod_{k=1}^{i_{0}} \mu^{(k)}(x) d x \\
& =\int_{[1, \infty)^{i_{0}}} \frac{\left(\min \left\{\|U(x)\|_{\alpha},\left\|V_{n}\right\|_{\beta}\right\}\right)^{\eta}}{\left(\max \left\{\|U(x)\|_{\alpha},\left\|V_{n}\right\|_{\beta}\right\}\right)^{\lambda+\eta}} \frac{\left\|V_{n}\right\|_{\beta}^{\lambda_{2}}}{\|U(x)\|_{\alpha}^{i_{0}-\lambda_{1}}} \prod_{k=1}^{i_{0}} \mu^{(k)}(x) d x \\
& \quad \geq \int_{[c, \infty)^{i_{0}}} \frac{\left(\min \left\{\|v\|_{\alpha},\left\|V_{n}\right\|_{\beta}\right\}\right)^{\eta}}{\left(\max \left\{\|v\|_{\alpha},\left\|V_{n}\right\|_{\beta}\right\}\right)^{\lambda+\eta}} \frac{\left\|V_{n}\right\|_{\beta}^{\lambda_{2}}}{\|v\|_{\alpha}^{i_{0}-\lambda_{1}}} d v .
\end{aligned}
$$

For $M>c i_{0}^{1 / \alpha}$, we set

$$
\Psi(u)= \begin{cases}0, & 0<u<\frac{c^{\alpha} i_{0}}{M^{\alpha}} \\ \frac{\left(\min \left\{M u^{1 / \alpha},\left\|V_{n}\right\|_{\beta}\right\}\right)^{\eta}}{\left(\max \left\{M u^{1 / \alpha},\left\|V_{n}\right\|_{\beta}\right\}\right)^{\lambda+\eta}} \frac{\left\|V_{n}\right\|_{\beta}^{\lambda_{2}}}{\left(M u^{1 / \alpha}\right)^{i_{0}-\lambda_{1}}}, & \frac{c^{\alpha} i_{0}}{M^{\alpha}} \leq u \leq 1 .\end{cases}
$$

By (12), it follows that

$$
\begin{aligned}
& \int_{\left\{x \in \mathbb{R}_{+}^{i_{0}} ; x_{i} \geq c\right\}} \frac{\left(\min \left\{\|x\|_{\alpha},\left\|V_{n}\right\|_{\beta}\right\}\right)^{\eta}}{\left(\max \left\{\|x\|_{\alpha},\left\|V_{n}\right\|_{\beta}\right\}\right)^{\lambda+\eta}} \frac{\left\|V_{n}\right\|_{\beta}^{\lambda_{2}}}{\|x\|_{\alpha}^{i_{0}-\lambda_{1}}} d x \\
& =\lim _{M \rightarrow \infty} \int \cdots \int_{D_{M}} \Psi\left(\sum_{i=1}^{i_{0}}\left(\frac{x_{i}}{M}\right)^{\alpha}\right) d x_{1} \cdots d x_{i_{0}} \\
& =\lim _{M \rightarrow \infty} \frac{M^{i_{0}} \Gamma^{i_{0}}\left(\frac{1}{\alpha}\right)}{\alpha^{i_{0}} \Gamma\left(\frac{i_{0}}{\alpha}\right)} \int_{c^{\alpha} i_{0} / M^{\alpha}}^{1} \frac{\left(\min \left\{M u^{\frac{1}{\alpha}},\left\|V_{n}\right\|_{\beta}\right\}\right)^{\eta}}{\left(\max \left\{M u^{\frac{1}{\alpha}},\left\|V_{n}\right\|_{\beta}\right\}\right)^{\lambda+\eta}} \frac{\left\|V_{n}\right\|_{\beta}^{\lambda_{2}} u^{\frac{i_{0}}{\alpha}-1} d u}{\left(M u^{\frac{1}{\alpha}}\right)^{i_{0}-\lambda_{1}}} \\
& \stackrel{\nu=\frac{M u^{1 / \alpha}}{\left\|V_{n}\right\|_{\beta}}}{=} \frac{\Gamma^{i_{0}}\left(\frac{1}{\alpha}\right)}{\alpha^{i_{0}-1} \Gamma\left(\frac{i_{0}}{\alpha}\right)} \int_{c i_{0}^{1 / \alpha} /\left\|V_{n}\right\|_{\beta}}^{1} \frac{(\min \{v, 1\})^{\eta} v^{\lambda_{1}-1}}{(\max \{v, 1\})^{\lambda+\eta}} d v .
\end{aligned}
$$

Hence, we have

$$
\begin{aligned}
w\left(\lambda_{1}, n\right) & >\frac{\Gamma^{i_{0}}\left(\frac{1}{\alpha}\right)}{\alpha^{i_{0}-1} \Gamma\left(\frac{i_{0}}{\alpha}\right)} \int_{c i_{0}^{1 / \alpha} /\left\|V_{n}\right\|_{\beta}}^{1} \frac{(\min \{v, 1\})^{\eta} v^{\lambda_{1}-1}}{(\max \{v, 1\})^{\lambda+\eta}} d v \\
& =K_{2}\left(\lambda_{1}\right)\left(1-\theta_{\lambda}(n)\right)>0 .
\end{aligned}
$$

For $\left\|V_{n}\right\|_{\beta} \geq c i_{0}^{1 / \alpha}$, we obtain

$$
\begin{aligned}
0 & <\theta_{\lambda}(n)=\frac{1}{k\left(\lambda_{1}\right)} \int_{0}^{c i_{0}^{1 / \alpha} /\left\|V_{n}\right\|_{\beta}} \frac{(\min \{v, 1\})^{\eta} v^{\lambda_{1}-1}}{(\max \{v, 1\})^{\lambda+\eta}} d v \\
& =\frac{1}{k\left(\lambda_{1}\right)} \int_{0}^{c i_{0}^{1 / \alpha} /\left\|V_{n}\right\|_{\beta}} v^{\lambda_{1}+\eta-1} d v=\frac{1}{\left(\lambda_{1}+\eta\right) k\left(\lambda_{1}\right)}\left(\frac{c i_{0}^{1 / \alpha}}{\left\|V_{n}\right\|_{\beta}}\right)^{\lambda_{1}+\eta},
\end{aligned}
$$

and then (21) and (22) follow. 


\section{Main results}

Setting functions

$$
\begin{aligned}
& \Phi(m):=\frac{\left\|U_{m}\right\|_{\alpha}^{p\left(i_{0}-\lambda_{1}\right)-i_{0}}}{\left(\prod_{k=1}^{i_{0}} \mu_{m}^{(k)}\right)^{p-1}} \quad\left(m \in \mathbb{N}^{i_{0}}\right), \\
& \Psi(n):=\frac{\left\|V_{n}\right\|_{\beta}^{q\left(j_{0}-\lambda_{2}\right)-j_{0}}}{\left(\prod_{l=1}^{j_{0}} v_{n}^{(l)}\right)^{q-1}} \quad\left(n \in \mathbb{N}^{j_{0}}\right),
\end{aligned}
$$

and the following normed spaces:

$$
\begin{aligned}
& l_{p, \Phi}:=\left\{a=\left\{a_{m}\right\} ;\|a\|_{p, \Phi}:=\left\{\sum_{m} \Phi(m)\left|a_{m}\right|^{p}\right\}^{\frac{1}{p}}<\infty\right\}, \\
& l_{q, \Psi}:=\left\{b=\left\{b_{n}\right\} ;\|b\|_{q, \Psi}:=\left\{\sum_{n} \Psi(n)\left|b_{n}\right|^{q}\right\}^{\frac{1}{q}}<\infty\right\}, \\
& l_{p, \Psi 1-p}:=\left\{c=\left\{c_{n}\right\} ;\|c\|_{p, \Psi 1-p}:=\left\{\sum_{n} \Psi^{1-p}(n)\left|c_{n}\right|^{p}\right\}^{\frac{1}{p}}<\infty\right\},
\end{aligned}
$$

we have the following.

Theorem 1 If $p>1, \frac{1}{p}+\frac{1}{q}=1, \alpha, \beta>0, \lambda>0,0<\lambda_{1}+\eta \leq i_{0}, 0<\lambda_{2}+\eta \leq j_{0}, \lambda_{1}+\lambda_{2}=\lambda$, then for $a_{m}, b_{n} \geq 0, a=\left\{a_{m}\right\} \in l_{p, \Phi}, b=\left\{b_{n}\right\} \in l_{q, \Psi},\|a\|_{p, \Phi},\|b\|_{q, \Psi}>0$, we have the following equivalent inequalities:

$$
\begin{aligned}
I & :=\sum_{n} \sum_{m} \frac{\left(\min \left\{\left\|U_{m}\right\|_{\alpha},\left\|V_{n}\right\|_{\beta}\right\}\right)^{\eta}}{\left(\max \left\{\left\|U_{m}\right\|_{\alpha},\left\|V_{n}\right\|_{\beta}\right\}\right)^{\lambda+\eta}} a_{m} b_{n}<K_{1}^{\frac{1}{p}}\left(\lambda_{1}\right) K_{2}^{\frac{1}{q}}\left(\lambda_{1}\right)\|a\|_{p, \Phi}\|b\|_{q, \Psi}, \\
J & :=\left\{\sum_{n} \frac{\prod_{k=1}^{j_{0}} v_{n}^{(k)}}{\left\|V_{n}\right\|_{\beta}^{j_{0}-p \lambda_{2}}}\left[\sum_{m} \frac{\left(\min \left\{\left\|U_{m}\right\|_{\alpha},\left\|V_{n}\right\|_{\beta}\right\}\right)^{\eta} a_{m}}{\left(\max \left\{\left\|U_{m}\right\|_{\alpha},\left\|V_{n}\right\|_{\beta}\right\}\right)^{\lambda+\eta}}\right]^{p}\right\}^{\frac{1}{p}} \\
& <K_{1}^{\frac{1}{p}}\left(\lambda_{1}\right) K_{2}^{\frac{1}{q}}\left(\lambda_{1}\right)\|a\|_{p, \Phi},
\end{aligned}
$$

where

$$
K_{1}^{\frac{1}{p}}\left(\lambda_{1}\right) K_{2}^{\frac{1}{q}}\left(\lambda_{1}\right)=\left[\frac{\Gamma^{j_{0}}\left(\frac{1}{\beta}\right)}{\beta^{j_{0}-1} \Gamma\left(\frac{j_{0}}{\beta}\right)}\right]^{\frac{1}{p}}\left[\frac{\Gamma^{i_{0}}\left(\frac{1}{\alpha}\right)}{\alpha^{i_{0}-1} \Gamma\left(\frac{i_{0}}{\alpha}\right)}\right]^{\frac{1}{q}} k\left(\lambda_{1}\right) .
$$

Proof By Hölder's inequality with weight (cf. [27]), we have

$$
\begin{aligned}
I= & \sum_{n} \sum_{m} \frac{\left(\min \left\{\left\|U_{m}\right\|_{\alpha},\left\|V_{n}\right\|_{\beta}\right\}\right)^{\eta}}{\left(\max \left\{\left\|U_{m}\right\|_{\alpha},\left\|V_{n}\right\|_{\beta}\right\}\right)^{\lambda+\eta}} \\
& \times\left[\frac{\left\|U_{m}\right\|_{\alpha}^{\frac{i_{0}-\lambda_{1}}{q}}}{\left\|V_{n}\right\|_{\beta}^{\frac{j_{0}-\lambda_{2}}{p}}} \frac{\left(\prod_{l=1}^{j_{0}} v_{n}^{(l)}\right)^{\frac{1}{p}} a_{m}}{\left(\prod_{k=1}^{i_{0}} \mu_{m}^{(k)}\right)^{\frac{1}{q}}}\right]\left[\frac{\left\|V_{n}\right\|_{\beta}^{\frac{j_{0}-\lambda_{2}}{p}}}{\left\|U_{m}\right\|_{\alpha}^{\frac{i_{0}-\lambda_{1}}{q}}} \frac{\left(\prod_{k=1}^{i_{0}} \mu_{m}^{(k)}\right)^{\frac{1}{q}} b_{n}}{\left(\prod_{l=1}^{j_{0}} v_{n}^{(l)}\right)^{\frac{1}{p}}}\right] \\
\leq & {\left[\sum_{m} W\left(\lambda_{2}, m\right) \frac{\left\|U_{m}\right\|_{\alpha}^{p\left(i_{0}-\lambda_{1}\right)-i_{0}} a_{m}^{p}}{\left(\prod_{k=1}^{i_{0}} \mu_{m}^{(k)}\right)^{p-1}}\right]^{\frac{1}{p}}\left[\sum_{n} w\left(\lambda_{1}, n\right) \frac{\left\|V_{n}\right\|_{\beta}^{q\left(j_{0}-\lambda_{2}\right)-j_{0}} b_{n}^{q}}{\left(\prod_{l=1}^{j_{0}} v_{n}^{(l)}\right)^{q-1}}\right]^{\frac{1}{q}} . }
\end{aligned}
$$


Then by (18) and (19), we have (23). We set

$$
b_{n}:=\frac{\prod_{l=1}^{j_{0}} v_{n}^{(l)}}{\left\|V_{n}\right\|_{\beta}^{j_{0}-p \lambda_{2}}}\left[\sum_{m} \frac{\left(\min \left\{\left\|U_{m}\right\|_{\alpha},\left\|V_{n}\right\|_{\beta}\right\}\right)^{\eta} a_{m}}{\left(\max \left\{\left\|U_{m}\right\|_{\alpha},\left\|V_{n}\right\|_{\beta}\right\}\right)^{\lambda+\eta}}\right]^{p-1}, \quad n \in \mathbb{N}^{j_{0}} .
$$

Then we have $J=\|b\|_{q, \Psi}^{q-1}$. Since the right-hand side of (24) is finite, it follows $J<\infty$. If $J=0$, then (24) is trivially valid; if $J>0$, then by (23), we have

$$
\begin{aligned}
& \|b\|_{q, \Psi}^{q}=J^{p}=I<K_{1}^{\frac{1}{p}}\left(\lambda_{1}\right) K_{2}^{\frac{1}{q}}\left(\lambda_{1}\right)\|a\|_{p, \Phi}\|b\|_{q, \Psi}, \\
& \|b\|_{q, \Psi}^{q-1}=J<K_{1}^{\frac{1}{p}}\left(\lambda_{1}\right) K_{2}^{\frac{1}{q}}\left(\lambda_{1}\right)\|a\|_{p, \Phi},
\end{aligned}
$$

namely (24) follows.

On the other hand, assuming that (24) is valid, by Hölder's inequality (cf. [27]), we have

$$
\begin{aligned}
I= & \sum_{n} \frac{\left(\prod_{l=1}^{j_{0}} v_{n}^{(l)}\right)^{1 / p}}{\left\|V_{n}\right\|_{\beta}^{\left(j_{0} / p\right)-\lambda_{2}}} \sum_{m} \frac{\left(\min \left\{\left\|U_{m}\right\|_{\alpha},\left\|V_{n}\right\|_{\beta}\right\}\right)^{\eta}}{\left(\max \left\{\left\|U_{m}\right\|_{\alpha},\left\|V_{n}\right\|_{\beta}\right\}\right)^{\lambda+\eta}} a_{m} \\
& \times \frac{\left\|V_{n}\right\|_{\beta}^{\left(j_{0} / p\right)-\lambda_{2}}}{\left(\prod_{l=1}^{j_{0}} v_{n}^{(l)}\right)^{1 / p}} b_{n} \leq J\|b\|_{q, \Psi} .
\end{aligned}
$$

Then by (24) we have (23), which is equivalent to (24).

Theorem 2 With regard to the assumptions of Theorem 1 , if $\mu_{m}^{(k)} \geq \mu_{m+1}^{(k)}(m \in \mathbb{N})$, $v_{n}^{(l)} \geq v_{n+1}^{(l)}(n \in \mathbb{N}), U_{\infty}^{(k)}=V_{\infty}^{(l)}=\infty\left(k=1, \ldots, i_{0}, l=1, \ldots, j_{0}\right)$, then the constant factor $K_{1}^{\frac{1}{p}}\left(\lambda_{1}\right) K_{2}^{\frac{1}{q}}\left(\lambda_{1}\right)$ in (23) and (24) is the best possible.

Proof For $0<\varepsilon<p\left(\lambda_{1}+\eta\right), \tilde{\lambda}_{1}=\lambda_{1}-\frac{\varepsilon}{p}\left(\in\left(-\eta,-\eta+i_{0}\right)\right), \tilde{\lambda}_{2}=\lambda_{2}+\frac{\varepsilon}{p}(>-\eta)$, we set

$$
\begin{array}{ll}
\tilde{a}=\left\{\tilde{a}_{m}\right\}, & \tilde{a}_{m}:=\left\|U_{m}\right\|_{\alpha}^{-i_{0}+\tilde{\lambda}_{1}} \prod_{k=1}^{i_{0}} \mu_{m}^{(k)} \quad\left(m \in \mathbb{N}^{i_{0}}\right), \\
\tilde{b}=\left\{\tilde{b}_{n}\right\}, \quad \tilde{b}_{n}:=\left\|V_{n}\right\|_{\beta}^{-j_{0}+\tilde{\lambda}_{2}-\varepsilon} \prod_{l=1}^{j_{0}} v_{n}^{(l)} \quad\left(n \in \mathbb{N}^{j_{0}}\right) .
\end{array}
$$

Then by (13) and (14), we obtain

$$
\begin{aligned}
\|\tilde{a}\|_{p, \Phi}\|\tilde{b}\|_{q, \Psi} & =\left[\sum_{m} \frac{\left\|U_{m}\right\|_{\alpha}^{p\left(i_{0}-\lambda_{1}\right)-i_{0}} \tilde{a}_{m}^{p}}{\left(\prod_{k=1}^{i_{0}} \mu_{m}^{(k)}\right)^{p-1}}\right]^{\frac{1}{p}}\left[\sum_{n} \frac{\left\|V_{n}\right\|_{\beta}^{q\left(j_{0}-\lambda_{2}\right)-j_{0}} \tilde{b}_{n}^{q}}{\left(\prod_{l=1}^{j_{0}} v_{n}^{(l)}\right)^{q-1}}\right]^{\frac{1}{q}} \\
& =\left(\sum_{m}\left\|U_{m}\right\|_{\alpha}^{-i_{0}-\varepsilon} \prod_{k=1}^{i_{0}} \mu_{m}^{(k)}\right)^{\frac{1}{p}}\left(\sum_{n}\left\|V_{n}\right\|_{\beta}^{-j_{0}-\varepsilon} \prod_{l=1}^{j_{0}} v_{n}^{(l)}\right)^{\frac{1}{q}} \\
& \leq \frac{1}{\varepsilon}\left(\frac{\Gamma^{i_{0}}\left(\frac{1}{\alpha}\right)}{i_{0}^{\varepsilon / \alpha} \alpha^{i_{0}-1} \Gamma\left(\frac{i_{0}}{\alpha}\right)}+\varepsilon O(1)\right)^{\frac{1}{p}}\left(\frac{\Gamma^{j_{0}}\left(\frac{1}{\beta}\right)}{j_{0}^{\varepsilon / \beta} \beta^{j_{0}-1} \Gamma\left(\frac{j_{0}}{\beta}\right)}+\varepsilon \tilde{O}(1)\right)^{\frac{1}{q}} .
\end{aligned}
$$


By (21) and (22), we find

$$
\begin{aligned}
\tilde{I} & :=\sum_{n}\left[\sum_{m} \frac{\left(\min \left\{\left\|U_{m}\right\|_{\alpha},\left\|V_{n}\right\|_{\beta}\right\}\right)^{\eta}}{\left(\max \left\{\left\|U_{m}\right\|_{\alpha},\left\|V_{n}\right\|_{\beta}\right\}\right)^{\lambda+\eta}} \tilde{a}_{m}\right] \tilde{b}_{n} \\
& =\sum_{n} w\left(\tilde{\lambda}_{1}, n\right)\left\|V_{n}\right\|_{\beta}^{-j_{0}-\varepsilon} \prod_{l=1}^{j_{0}} v_{n}^{(l)} \\
& >K_{2}\left(\tilde{\lambda}_{1}\right) \sum_{n}\left(1-O\left(\frac{1}{\left\|V_{n}\right\|_{\beta}^{\lambda_{1}+\eta}}\right)\right)\left\|V_{n}\right\|_{\beta}^{-j_{0}-\varepsilon} \prod_{l=1}^{j_{0}} v_{n}^{(l)} \\
& =K_{2}\left(\tilde{\lambda}_{1}\right)\left(\frac{\Gamma^{j_{0}}\left(\frac{1}{\beta}\right)}{\varepsilon j_{0}^{\varepsilon / \beta} \beta^{j_{0}-1} \Gamma\left(\frac{j_{0}}{\beta}\right)}+\tilde{O}(1)-O_{1}(1)\right) .
\end{aligned}
$$

If there exists a constant $K \leq K_{1}^{\frac{1}{p}}\left(\lambda_{1}\right) K_{2}^{\frac{1}{q}}\left(\lambda_{1}\right)$ such that (23) is valid when replacing $K_{1}^{\frac{1}{p}}\left(\lambda_{1}\right) K_{2}^{\frac{1}{q}}\left(\lambda_{1}\right)$ by $K$, then we have $\varepsilon \tilde{I}<\varepsilon K\|\tilde{a}\|_{p, \Phi}\|\tilde{b}\|_{q, \Psi}$, namely

$$
\begin{aligned}
& K_{2}\left(\lambda_{1}-\frac{\varepsilon}{p}\right)\left(\frac{\Gamma^{j_{0}}\left(\frac{1}{\beta}\right)}{j_{0}^{\varepsilon / \beta} \beta^{j_{0}-1} \Gamma\left(\frac{j_{0}}{\beta}\right)}+\varepsilon \tilde{O}(1)-\varepsilon O_{1}(1)\right) \\
& \quad<K\left(\frac{\Gamma^{i_{0}}\left(\frac{1}{\alpha}\right)}{i_{0}^{\varepsilon / \alpha} \alpha^{i_{0}-1} \Gamma\left(\frac{i_{0}}{\alpha}\right)}+\varepsilon O(1)\right)^{\frac{1}{p}}\left(\frac{\Gamma^{j_{0}}\left(\frac{1}{\beta}\right)}{j_{0}^{\varepsilon / \beta} \beta^{j_{0}-1} \Gamma\left(\frac{j_{0}}{\beta}\right)}+\varepsilon \tilde{O}(1)\right)^{\frac{1}{q}} .
\end{aligned}
$$

For $\varepsilon \rightarrow 0^{+}$, we find

$$
\frac{\Gamma^{j_{0}}\left(\frac{1}{\beta}\right)}{\beta^{j_{0}-1} \Gamma\left(\frac{j_{0}}{\beta}\right)} \frac{\Gamma^{i_{0}}\left(\frac{1}{\alpha}\right) k\left(\lambda_{1}\right)}{\alpha^{i_{0}-1} \Gamma\left(\frac{i_{0}}{\alpha}\right)} \leq K\left[\frac{\Gamma^{i_{0}}\left(\frac{1}{\alpha}\right)}{\alpha^{i_{0}-1} \Gamma\left(\frac{i_{0}}{\alpha}\right)}\right]^{\frac{1}{p}}\left[\frac{\Gamma^{j_{0}}\left(\frac{1}{\beta}\right)}{\beta^{j_{0}-1} \Gamma\left(\frac{j_{0}}{\beta}\right)}\right]^{\frac{1}{q}}
$$

and then $K_{1}^{\frac{1}{p}}\left(\lambda_{1}\right) K_{2}^{\frac{1}{q}}\left(\lambda_{1}\right) \leq K$. Hence, $K=K_{1}^{\frac{1}{p}}\left(\lambda_{1}\right) K_{2}^{\frac{1}{q}}\left(\lambda_{1}\right)$ is the best possible constant factor of (23). The constant factor in (24) is still the best possible. Otherwise, we would reach a contradiction by (26) that the constant factor in (23) is not the best possible.

\section{Operator expressions}

With regard to the assumptions of Theorem 2, in view of

$$
\begin{aligned}
& c_{n}:=\frac{\prod_{k=1}^{j_{0}} v_{n}^{(k)}}{\left\|V_{n}\right\|_{\beta}^{j_{0}-p \lambda_{2}}}\left[\sum_{m} \frac{\left(\min \left\{\left\|U_{m}\right\|_{\alpha},\left\|V_{n}\right\|_{\beta}\right\}\right)^{\eta}}{\left(\max \left\{\left\|U_{m}\right\|_{\alpha},\left\|V_{n}\right\|_{\beta}\right\}\right)^{\lambda+\eta}} a_{m}\right]^{p-1}, \quad n \in \mathbb{N}^{j_{0}}, \\
& c=\left\{c_{n}\right\}, \quad\|c\|_{p, \Psi 1-p}=J<K_{1}^{\frac{1}{p}}\left(\lambda_{1}\right) K_{2}^{\frac{1}{q}}\left(\lambda_{1}\right)\|a\|_{p, \Phi}<\infty,
\end{aligned}
$$

we can set the following definition.

Definition 2 Define a multidimensional Hilbert's operator $T: l_{p, \Phi} \rightarrow l_{p, \Psi^{1-p}}$ as follows: For any $a \in l_{p, \Phi}$, there exists a unique representation $T a=c \in l_{p, \Psi^{1-p}}$, satisfying

$$
\operatorname{Ta}(n):=\sum_{m} \frac{\left(\min \left\{\left\|U_{m}\right\|_{\alpha},\left\|V_{n}\right\|_{\beta}\right\}\right)^{\eta}}{\left(\max \left\{\left\|U_{m}\right\|_{\alpha},\left\|V_{n}\right\|_{\beta}\right\}\right)^{\lambda+\eta}} a_{m} \quad\left(n \in \mathbb{N}^{j 0}\right) .
$$


For $b \in l_{q, \Psi}$, we define the following formal inner product of $T a$ and $b$ as follows:

$$
(T a, b):=\sum_{n}\left[\sum_{m} \frac{\left(\min \left\{\left\|U_{m}\right\|_{\alpha},\left\|V_{n}\right\|_{\beta}\right\}\right)^{\eta}}{\left(\max \left\{\left\|U_{m}\right\|_{\alpha},\left\|V_{n}\right\|_{\beta}\right\}\right)^{\lambda+\eta}} a_{m}\right] b_{n} .
$$

Then by Theorems 1 and 2, we have the following equivalent inequalities:

$$
\begin{aligned}
& (T a, b)<K_{1}^{\frac{1}{p}}\left(\lambda_{1}\right) K_{2}^{\frac{1}{q}}\left(\lambda_{1}\right)\|a\|_{p, \Phi}\|b\|_{q, \Psi}, \\
& \|T a\|_{p, \Psi^{1-p}}<K_{1}^{\frac{1}{p}}\left(\lambda_{1}\right) K_{2}^{\frac{1}{q}}\left(\lambda_{1}\right)\|a\|_{p, \Phi} .
\end{aligned}
$$

It follows that $T$ is bounded with

$$
\|T\|:=\sup _{a(\neq \theta) \in l_{p, \Phi}} \frac{\|T a\|_{p, \Psi 1-p}}{\|a\|_{p, \Phi}} \leq K_{1}^{\frac{1}{p}}\left(\lambda_{1}\right) K_{2}^{\frac{1}{q}}\left(\lambda_{1}\right) .
$$

Since the constant factor $K_{1}^{\frac{1}{p}}\left(\lambda_{1}\right) K_{2}^{\frac{1}{q}}\left(\lambda_{1}\right)$ in (30) is the best possible, we have

$$
\|T\|=K_{1}^{\frac{1}{p}}\left(\lambda_{1}\right) K_{2}^{\frac{1}{q}}\left(\lambda_{1}\right)=\left[\frac{\Gamma^{j_{0}}\left(\frac{1}{\beta}\right)}{\beta^{j_{0}-1} \Gamma\left(\frac{j_{0}}{\beta}\right)}\right]^{\frac{1}{p}}\left[\frac{\Gamma^{i_{0}}\left(\frac{1}{\alpha}\right)}{a^{i_{0}-1} \Gamma\left(\frac{i_{0}}{\alpha}\right)}\right]^{\frac{1}{q}} k\left(\lambda_{1}\right) .
$$

Remark 1 (i) For $\mu_{i}=v_{j}=1(i, j \in \mathbb{N}$ ), (23) reduces to (4). Hence, (23) is an extension of (4).

(ii) For $\eta=0,0<\lambda_{1} \leq i_{0}, 0<\lambda_{2} \leq j_{0}$, (23) reduces to the following inequality:

$$
\begin{aligned}
\sum_{n} & \sum_{m} \frac{1}{\left(\max \left\{\left\|U_{m}\right\|_{\alpha},\left\|V_{n}\right\|_{\beta}\right\}\right)^{\lambda}} a_{m} b_{n} \\
< & \left.<\frac{\Gamma^{j_{0}}\left(\frac{1}{\beta}\right)}{\beta^{j 0^{-1}} \Gamma\left(\frac{j_{0}}{\beta}\right)}\right]^{\frac{1}{p}}\left[\frac{\Gamma^{i_{0}}\left(\frac{1}{\alpha}\right)}{a^{i_{0}-1} \Gamma\left(\frac{i_{0}}{\alpha}\right)}\right]^{\frac{1}{q}} \frac{\lambda}{\lambda_{1} \lambda_{2}}\|a\|_{p, \Phi}\|b\|_{q, \Psi} .
\end{aligned}
$$

In particular, for $i_{0}=j_{0}=\lambda=1, \lambda_{1}=\frac{1}{q}, \lambda_{2}=\frac{1}{p}$, (33) reduces to (5). Hence, (33) is also an extension of (5); so is (23).

(iii) For $\eta=-\lambda, \lambda_{1}, \lambda_{2}<0$, (23) reduces to the following inequality:

$$
\begin{aligned}
\sum_{n} & \sum_{m} \frac{1}{\left(\min \left\{\left\|U_{m}\right\|_{\alpha},\left\|V_{n}\right\|_{\beta}\right\}\right)^{\lambda}} a_{m} b_{n} \\
& <\left[\frac{\Gamma^{j_{0}}\left(\frac{1}{\beta}\right)}{\beta j^{j_{0}-1} \Gamma\left(\frac{j_{0}}{\beta}\right)}\right]^{\frac{1}{p}}\left[\frac{\Gamma^{i_{0}}\left(\frac{1}{\alpha}\right)}{a^{i_{0}-1} \Gamma\left(\frac{i_{0}}{\alpha}\right)}\right]^{\frac{1}{q}} \frac{(-\lambda)}{\lambda_{1} \lambda_{2}}\|a\|_{p, \Phi}\|b\|_{q, \Psi} .
\end{aligned}
$$

(iv) For $\lambda=0, \lambda_{2}=-\lambda_{1}\left(-\eta<\lambda_{1}<\eta\right)$, (23) reduces to the following inequality:

$$
\begin{aligned}
\sum_{n} & \sum_{m}\left(\frac{\min \left\{\left\|U_{m}\right\|_{\alpha},\left\|V_{n}\right\|_{\beta}\right\}}{\max \left\{\left\|U_{m}\right\|_{\alpha},\left\|V_{n}\right\|_{\beta}\right\}}\right)^{\eta} a_{m} b_{n} \\
< & {\left[\frac{\Gamma^{j_{0}}\left(\frac{1}{\beta}\right)}{\beta^{j_{0}-1} \Gamma\left(\frac{j_{0}}{\beta}\right)}\right]^{\frac{1}{p}}\left[\frac{\Gamma^{i_{0}}\left(\frac{1}{\alpha}\right)}{a^{i_{0}-1} \Gamma\left(\frac{i_{0}}{\alpha}\right)}\right]^{\frac{1}{q}} \frac{2 \eta}{\eta^{2}-\lambda_{1}^{2}}\|a\|_{p, \Phi}\|b\|_{q, \Psi} . }
\end{aligned}
$$

The above particular inequalities are also with the best possible constant factors. 


\section{Competing interests}

The authors declare that they have no competing interests.

\section{Authors' contributions}

BY carried out the mathematical studies, participated in the sequence alignment and drafted the manuscript. JZ participated in the design of the study and performed the numerical analysis. All authors read and approved the final manuscript.

\section{Acknowledgements}

This work is supported by the National Natural Science Foundation (No. 61370186, No. 61640222), and Appropriative Researching Fund for Professors and Doctors, Guangdong University of Education (No. 2015ARF25). We are grateful for their help.

\section{Publisher's Note}

Springer Nature remains neutral with regard to jurisdictional claims in published maps and institutional affiliations.

Received: 20 January 2017 Accepted: 23 March 2017 Published online: 18 April 2017

\section{References}

1. Hardy, GH, Littlewood, JE, Pólya, G: Inequalities. Cambridge University Press, Cambridge (1934)

2. Mitrinović, DS, Pečarić, JE, Fink, AM: Inequalities Involving Functions and Their Integrals and Derivatives. Kluwer Academic, Boston (1991)

3. Yang, BC: The Norm of Operator and Hilbert-Type Inequalities. Science Press, Beijin (2009) (in Chinese)

4. Yang, BC, Chen, Q: A multidimensional discrete Hilbert-type inequality. J. Math. Inequal. 8(2), 267-277 (2014)

5. Hong, Y: On Hardy-Hilbert integral inequalities with some parameters. J. Inequal. Pure Appl. Math. 6(4), Article ID 92 (2005)

6. Zhong, WY, Yang, BC: On multiple Hardy-Hilbert's integral inequality with kernel. J. Inequal. Appl. 2007, Article ID 27962 (2007)

7. Yang, BC, Krnić, M: On the norm of a multi-dimensional Hilbert-type operator. Sarajevo J. Math. 7(20), 223-243 (2011)

8. Krnić, M, Pečarić, JE, Vuković, P: On some higher-dimensional Hilbert's and Hardy-Hilbert's type integral inequalities with parameters. Math. Inequal. Appl. 11, 701-716 (2008)

9. Krnić, M, Vuković, P: On a multidimensional version of the Hilbert-type inequality. Anal. Math. 38, 291-303 (2012)

10. Rassias, M, Yang, BC: A multidimensional half-discrete Hilbert-type inequality and the Riemann zeta function. Appl. Math. Comput. 225, 263-277 (2013)

11. Yang, BC: A multidimensional discrete Hilbert-type inequality. Int. J. Nonlinear Anal. Appl. 5(1), 80-88 (2014)

12. Chen, Q, Yang, BC: On a more accurate multidimensional Mulholland-type inequality. J. Inequal. Appl. 2014,322 (2014)

13. Rassias, M, Yang, BC: On a multidimensional Hilbert-type integral inequality associated to the gamma function. Appl. Math. Comput. 249, 408-418 (2014)

14. Yang, BC: On a more accurate multidimensional Hilbert-type inequality with parameters. Math. Inequal. Appl. 18(2), 429-441 (2015)

15. Huang, ZX, Yang, BC: A multidimensional Hilbert-type integral inequality. J. Inequal. Appl. 2015, 151 (2015)

16. Liu, T, Yang, BC, He, LP: On a multidimensional Hilbert-type integral inequality with logarithm function. Math. Inequal. Appl. 18(4), 1219-1234 (2015)

17. Shi, YP, Yang, BC: On a multidimensional Hilbert-type inequality with parameters. J. Inequal. Appl. 2015,371 (2015)

18. Shi, YP, Yang, BC: A new Hardy-Hilbert-type inequality with multi-parameters and a best possible constant factor. J. Inequal. Appl. 2015, 380 (2015)

19. Huang, QL: A new extension of Hardy-Hilbert-type inequality. J. Inequal. Appl. 2015, 397 (2015)

20. Wang, AZ, Huang, QL, Yang, BC: A strengthened Mulholland-type inequality with parameters. J. Inequal. Appl. 2015, $329(2015)$

21. Yang, BC, Chen, Q: On a Hardy-Hilbert-type inequality with parameters. J. Inequal. Appl. 2015, 339 (2015)

22. Li, AH, Yang, BC, He, LP: On a new Hardy-Mulholland-type inequality and its more accurate form. J. Inequal. Appl. 2016, 69 (2016)

23. Rassias, M, Yang, BC: On a Hardy-Hilbert-type inequality with a general homogeneous kernel. Int. J. Nonlinear Anal. Appl. 7(1), 249-269 (2016)

24. Chen, Q, Shi, YP, Yang, BC: A relation between two simple Hardy-Mulholland-type inequalities with parameters. J. Inequal. Appl. 2016, 75 (2016)

25. Yang, BC, Chen, Q: On a more accurate Hardy-Mulholland-type inequality. J. Inequal. Appl. 2016, 82 (2016)

26. Yang, BC: Hilbert-type integral operators: norms and inequalities. In: Pardalos, PM, Georgiev, PG, Srivastava, HM (eds.) Nonlinear Analysis, Stability, Approximation, and Inequalities, pp. 771-859. Springer, New York (2012)

27. Kuang, JC: Applied Inequalities. Shangdong Science Technic Press, Jinan, China (2004) 